\title{
BABAR resonance as a new window of hadron physics
}

\author{
$\operatorname{AUTHOR}(S)$ :
}

Terasaki, $\mathrm{K}$

CITATION:

Terasaki, K. BABAR resonance as a new window of hadron physics. PHYSICAL REVIEW D 2003, 68(1): 011501.

ISSUE DATE:

2003-07

URL:

http://hdl.handle.net/2433/50463

RIGHT:

Copyright 2003 American Physical Society 
PHYSICAL REVIEW D 68, 011501(R) (2003)

\title{
BABAR resonance as a new window of hadron physics
}

\author{
K. Terasaki \\ Yukawa Institute for Theoretical Physics, Kyoto University, Kyoto 606-8502, Japan
}

(Received 21 May 2003; published 7 July 2003)

\begin{abstract}
The possible decays of four-quark mesons are studied by assigning the newly observed BABAR resonance to a four-quark meson, $\hat{F}_{I}^{+} \sim[c q][\bar{s} \bar{q}]$ with $q=u, d$. It is expected that some of them can be observed as narrow resonances. The implication of the existence of four-quark mesons in hadronic weak interactions is also discussed.
\end{abstract}

DOI: 10.1103/PhysRevD.68.011501

PACS number(s): 14.40.Lb, 13.25.Ft

Recently the BABAR Collaboration [1] has observed a narrow $D_{s}^{+} \pi^{0}$ resonance with a mass $2317.6 \pm 1.3 \mathrm{MeV}$ and a width $8.8 \pm 1.1 \mathrm{MeV}$ (a Gaussian fit but the intrinsic width $\$ 10 \mathrm{MeV}$ ), which has been confirmed by the CLEO Collaboration [2], and suggested that it is a scalar four-quark meson.

Four quark mesons $\{q q \bar{q} \bar{q}\}$ can be classified into four types [3]:

$$
\{q q \bar{q} \bar{q}\}=[q q][\bar{q} \bar{q}] \oplus(q q)(\bar{q} \bar{q}) \oplus\{[q q](\bar{q} \bar{q}) \pm(q q)[\bar{q} \bar{q}]\},
$$

where parentheses and square brackets denote symmetry and antisymmetry, respectively. The first two terms on the righthand-side of Eq. (1) can have $J^{P(C)}=0^{+(+)}$. Each of them is again classified into two classes because of two different ways to produce a color singlet $\{q q \bar{q} \bar{q}\}$. However, since these two can mix with each other, they are classified into heavier and lighter ones. We discriminate these two by putting * on the former as in Table I in accordance with Ref. [3] in which the four-quark mesons were studied within the framework of $q=u, d$ and $s$. To explore the hadronic weak decays of the charm mesons, we have extended the above framework straightforwardly to $q=u, d, s$ and $c$, and studied the role of four-quark mesons [4-6]. The heavier class of $[q q][\bar{q} \bar{q}]$ and $(q q)(\bar{q} \bar{q})($ with $*)$ can play a dramatic role in the charm decays while the lighter class (without*) can play an important role in the hadronic weak interactions of the $K$ mesons [7]. Not only in hadron spectroscopy but also in the hadronic weak interactions of the $K$ and charm mesons, therefore, it is very important to confirm the existence of four-quark mesons.

In this Rapid Communication, however, we will concentrate on the $[c q][\bar{q} \bar{q}]$ mesons (with $q=u, d, s$ ) since the other types of the four-quark mesons will be massive enough to decay into two pseudoscalar mesons and therefore they will be broad and not very easy to observe. In Ref. [6], however, the mass of $\hat{F}_{I} \sim[c q][\bar{s} \bar{q}]$ (with $q=u, d$ ), which was crudely estimated by using simple quark counting and by using the results on the light four-quark meson masses in Ref. [3], was higher by $\sim 100 \mathrm{MeV}$ than the measured one of the BABAR resonance since we took $\Delta m_{s}=m_{s}$ $-m_{u}=0.2 \mathrm{GeV}$. We here revise the mass values of the $[c q][\bar{q} \bar{q}]$ mesons exchanging the old value of $\Delta m_{s}$ by a new one, $\Delta m_{s} \simeq m_{D_{s}}-m_{D} \simeq 0.1 \mathrm{GeV}$ at $\sim 2 \mathrm{GeV}$ scale, and using the measured $m_{\hat{F}_{I}}=2.32 \mathrm{GeV}$ as the input data. (We will assign the BABAR resonance to $\hat{F}_{I}^{+}$later.) Their revised mass values are listed in Table I. (The notations have been given in Ref. [6]. $\hat{E}^{0} \sim[c s][\bar{u} \bar{d}]$ was previously described by $\hat{F}^{0}$ but it seems to be misleading so it is now revised. See also, for more details, Ref. [3].) To estimate more precisely the masses of the $[c q][\bar{q} \bar{q}]$ mesons with *, we need additional input data.

As seen in Table I, the four-quark mesons with * have large enough masses to decay into two pseudoscalar mesons so that they will be broad as mentioned before. On the contrary, the estimated masses of $[c q][\bar{q} \bar{q}]$ without $*$ are close to the thresholds of the two body decays through strong interactions. Therefore, some of them can decay through strong interactions but their rates will be rather small due to their small phase space, so that they will be observed as narrow resonances such as the BABAR resonance. Since some of them are not massive enough to decay into two pseudoscalar mesons through strong interactions, their dominant decays may be $I$-spin non-conserving ones (unless their masses are higher than the expected ones).

The $\hat{F}_{I}$ mesons form an iso-triplet, $\hat{F}_{I}^{++}, \hat{F}_{I}^{+}$and $\hat{F}_{I}^{0}$, where iso $(I)$-spin symmetry is always assumed in this paper unless we note in particular. Then all of them can have the same type of kinematically allowed decays, $\hat{F}_{I} \rightarrow D_{s}^{+} \pi$, with different charge states. The other decays, for example, $\hat{F}_{I}$ $\rightarrow D_{s}^{+}(\pi \pi)$, are not allowed kinematically.

We have two iso-doublets $\hat{D}$ and $\hat{D}^{s}$. The former can

TABLE I. Ideally mixed scalar $[c q][\bar{q} \bar{q}]$ mesons (with $q$ $=u, d, s)$, where $S$ and $I$ denote strangeness and $I$ spin.

\begin{tabular}{lcccc}
\hline \hline$S$ & $I=1$ & $I=\frac{1}{2}$ & $I=0$ & $\operatorname{Mass}(\mathrm{GeV})$ \\
\hline \multirow{3}{*}{1} & $\hat{F}_{I}$ & & $\hat{F}_{0}$ & $2.32^{\mathrm{a}}$ \\
& $\hat{F}_{I}^{*}$ & & $\hat{F}_{0}^{*}$ & $(3.1)$ \\
& & $\hat{D}$ & & 2.22 \\
& & $\hat{D}^{*}$ & & $(3.0)$ \\
& & $\hat{D}^{s}$ & & 2.42 \\
& & $\hat{D}^{s *}$ & & $(3.2)$ \\
& & & $\hat{E}^{0}$ & 2.32 \\
-1 & & $\hat{E}^{0 *}$ & $(3.10)$ \\
\hline \hline
\end{tabular}

anput data. 
decay into $D \pi$ final states, $\hat{D} \rightarrow D \pi$, and the kinematical condition is similar to the one in the decay, $\hat{F}_{I} \rightarrow D_{s}^{+} \pi$, as long as the mass value of $\hat{D}$ in Table I is taken. The latter contains an $s \bar{s}$ pair so that its decay mode is limited because of the Okubo-Zweig-Iizuka (OZI) rule [8]. One of the possible decays would be $\hat{D}^{s} \rightarrow D \eta^{s} \rightarrow D \eta$. However, since this decay is approximately on the threshold, i.e., $m_{\hat{D}^{s}} \simeq m_{D}$ $+m_{\eta}$, it is not clear if such a decay is allowed kinematically, as long as the estimated value of $m_{\hat{D}^{s}}$ in Table I is taken. Even if allowed, the rate would be very small because of the small phase space.

$\hat{F}_{0}^{+}$is an iso-singlet counterpart of the $\hat{F}_{I}^{+}$mesons. It cannot decay into $D_{s}^{+} \pi^{0}$ as long as the $I$ spin is conserved, so that it will decay dominantly through $I$-spin non-conserving interactions (such as electromagnetic interactions). In this case, the width of $\hat{F}_{0}^{+}$will be much narrower than that of the BABAR resonance. If its mass should be higher (by $\gtrsim 50 \mathrm{MeV}$ ) because of some $I$-spin dependent force, it could decay dominantly into the $D K$ final states and its width might reproduce the measured one of the BABAR resonance.

$\hat{E}^{0}$ is exotic. It is an iso-singlet scalar meson with a charm $C=1$ and a strangeness $S=-1$, i.e., $\hat{E}_{0}^{+} \sim[c s][\bar{u} \bar{d}]$. It cannot decay into the $D \bar{K}$ final states unless it is massive enough because of some extra force. If its mass is almost the same as the $\hat{F}_{0}$, then it still cannot decay through strong interactions or electromagnetic interactions $[9,10]$ as there are no ordinary mesons with $C=1$ and $S=-1$. Therefore, if it can be created, it will have a very long life.

Now we study numerically the decays of the $[c q][\bar{q} \bar{q}]$ mesons by assigning the BABAR resonance to $\hat{F}_{I}^{+}$although there exist many proposals to assign it to the other hadron states such as a $(D K)$ molecule [11] (or atom [12]), an isosinglet four-quark meson [13], an excited $(c \bar{s})$ state [14], a chiral partner of $D_{s}^{+}$[15], etc. (However, there are arguments which do not support the last two assignments $[16,17]$.) Consider, as an example, a decay, $A(\mathbf{p}) \rightarrow B\left(\mathbf{p}^{\prime}\right)+\pi(\mathbf{q})$, where $A, B$ and $\pi$ are a parent, a daughter pseudoscalar and a $\pi$ meson, respectively. The rate for the decay is given by

$$
\Gamma(A \rightarrow B \pi)=\frac{1}{2 J_{A}+1} \frac{q_{c}}{8 \pi m_{A}^{2}} \sum_{\text {spin }}|M(A \rightarrow B \pi)|^{2},
$$

where $J_{A}, q_{c}$ and $M(A \rightarrow B \pi)$ denote the spin of $A$, the center-of-mass momentum of the final mesons and the decay amplitude, respectively. To calculate the amplitude, we here use the partially conserved axial-vector current (PCAC) hypothesis and a hard pion approximation in the infinite momentum frame (IMF), i.e., $\mathbf{p} \rightarrow \infty$ [18]. In this approximation, the amplitude is evaluated at a slightly unphysical point, i.e., $m_{\pi}^{2} \rightarrow 0$. In this way, the amplitude is given approximately by

$$
M(A \rightarrow B \pi)=\left(\frac{m_{A}^{2}-m_{B}^{2}}{f_{\pi}}\right)\langle B|A \bar{\pi}| A\rangle,
$$

where $A_{\pi}$ is the axial counterpart of the $I$ spin.
TABLE II. The assumed dominant decays of the scalar $[c q][\bar{q} \bar{q}]$ mesons and their estimated widths. $\Gamma\left(\hat{F}_{I}^{+} \rightarrow D_{s}^{+} \pi^{0}\right)$ $=8.8 \mathrm{MeV}$ is used as the input data. The decays into the final states between the angular brackets are not allowed kinematically as long as the parent mass values in the parentheses are taken.

\begin{tabular}{lcc}
\hline \hline $\begin{array}{l}\text { Parent } \\
\text { Mass in GeV) }\end{array}$ & Final state & Width $(\mathrm{MeV})$ \\
\hline$\hat{F}_{I}^{++}(2.32)$ & $D_{s}^{+} \pi^{+}$ & \\
$\hat{F}_{I}^{+}(2.32)$ & $D_{s}^{+} \pi^{0}$ & 8.8 \\
$\hat{F}_{I}^{0}(2.32)$ & $D_{s}^{+} \pi^{-}$ & \\
$\hat{D}^{+}(2.22)$ & $D^{0} \pi^{+}$ & 9.0 \\
$\hat{D}^{0}(2.22)$ & $D^{+} \pi^{0}$ & 4.5 \\
& $D^{+} \pi^{-}$ & 9.0 \\
$\hat{D}^{s}(2.42)$ & $D^{0} \pi^{0}$ & 4.5 \\
$\hat{F}_{0}^{+}(2.32)$ & $D \eta$ & $(I$-spin violation $)$ \\
$\hat{E}^{0}(2.32)$ & $\left\langle D_{s}^{+} \eta\right\rangle$ & \\
\hline \hline
\end{tabular}

Asymptotic matrix elements (matrix elements taken between the single hadron states with infinite momentum) of $A_{\pi}$ can be parametrized by using asymptotic flavor symmetry (flavor symmetry of the asymptotic matrix elements). (Asymptotic symmetry and its fruitful results were reviewed in Ref. [18].) Asymptotic matrix elements including the fourquark states have been parametrized previously [4-6]. We here list the related ones:

$$
\begin{aligned}
\left\langle D_{s}^{+}\left|A_{\pi^{-}}\right| \hat{F}_{I}^{++}\right\rangle & =\sqrt{2}\left\langle D_{s}^{+}\left|A_{\pi^{0}}\right| \hat{F}_{I}^{+}\right\rangle=\left\langle D_{s}^{+}\left|A_{\pi^{+}}\right| \hat{F}_{I}^{0}\right\rangle \\
& =-\left\langle D^{0}\left|A_{\pi^{-}}\right| \hat{D}^{+}\right\rangle=2\left\langle D^{+}\left|A_{\pi^{0}}\right| \hat{D}^{+}\right\rangle \\
& =-2\left\langle D^{0}\left|A_{\pi^{0}}\right| \hat{D}^{0}\right\rangle=-\left\langle D^{+}\left|A_{\pi^{+}}\right| \hat{D}^{0}\right\rangle .
\end{aligned}
$$

Inserting Eq. (3) with Eq. (4) into Eq. (2), we can calculate the approximate rates for the allowed two-body decays mentioned before. Here we equate the calculated width for the $\hat{F}_{I}^{+} \rightarrow D_{s}^{+} \pi^{0}$ decay to the measured one of the BABAR resonance, i.e.,

$$
\Gamma\left(\hat{F}_{I}^{+} \rightarrow D_{s}^{+} \pi^{0}\right) \simeq 8.8 \mathrm{MeV},
$$

since we do not find any other decays which can have large rates, and use it as the input data when we estimate the rates for the other decays. The results are listed in Table II. All the calculated widths of $\hat{F}_{I}$ and $\hat{D}$ are lying in the region 4.5-9.0 $\mathrm{MeV}$ so that they will be observed as narrow resonances in the $D_{s}^{+} \pi$ and $D \pi$ channels, respectively. The $\hat{D}^{s} \rightarrow D \eta$ decays are approximately on the threshold $m_{\hat{D}^{s}} \simeq m_{D}+m_{\eta}$ so that it is not clear if they are kinematically allowed. Besides, the decay is sensitive to the $\eta$ - $\eta^{\prime}$ mixing scheme which is still model dependent [19]. Therefore, we need more precise 
and reliable values of $m_{\hat{D}^{s}}, \eta$ - $\eta^{\prime}$ mixing parameters and decay constants in the $\eta-\eta^{\prime}$ system to obtain a definite result.

In summary we have studied the decays of the scalar $[c q][\bar{q} \bar{q}]$ mesons into two pseudoscalar mesons by assigning the BABAR resonance to $\hat{F}_{I}^{+}$(in our notation) and assuming the $I$-spin conservation. All the allowed decays are not very far from the corresponding thresholds so that their rates have been expected to saturate approximately their total widths. Therefore, we have used the measured width as the input data, $\Gamma\left(\hat{F}_{I}^{+} \rightarrow D_{s}^{+} \pi^{0}\right) \simeq 8.8 \mathrm{MeV} . \hat{F}_{I}$ and $\hat{D}$ could be observed as narrow resonances such as the BABAR observation. It is very much different from the results in Ref. [13] in which $\widetilde{D}_{0 s}\left(\hat{F}_{0}^{+}\right.$in our notation) was assigned to the BABAR resonance and all the other $[c \bar{q} q \bar{q}]$ mesons were predicted to be much broader ( $\sim 100 \mathrm{MeV}$ or more). To distinguish the present assignment from the other models and to confirm it, therefore, it is important to observe these narrow resonances.

We have not studied numerically the $\hat{D}^{s} \rightarrow D \eta$. Since the decay is approximately on the threshold and sensitive to the model dependent $\eta$ - $\eta^{\prime}$ mixing, it is hard to obtain a definite result on this decay at the present stage, although we can qualitatively expect that $\hat{D}^{s}$ will be much narrower than $\hat{F}_{I}$ and $\hat{D}$.

$\hat{E}^{0}$ will decay through weak interactions if it is created as long as its mass is below the $\hat{E}^{0} \rightarrow D \bar{K}$ threshold.
We have studied, so far, the strong decay properties of a group of the four-quark mesons. If their existence is confirmed, it will be very helpful in the understanding of the hadronic weak decays of the $K$ and charm mesons. The existence of the $[q q][\bar{q} \bar{q}]$ and $(q q)(\bar{q} \bar{q})$ mesons (with *) immediately leads to a solution to the long standing puzzle in the charm decays [20],

$$
\frac{\Gamma\left(D^{0} \rightarrow K^{+} K^{-}\right)}{\Gamma\left(D^{0} \rightarrow \pi^{+} \pi^{-}\right)} \simeq 3
$$

consistently with the other two-body decays of the charm mesons [4-6]. Furthermore, the lighter $(q q)(\bar{q} \bar{q})$ (without *) are useful in understanding the $|\Delta \mathbf{I}|=\frac{1}{2}$ rule and its violation in the $K \rightarrow \pi \pi$ decays consistently with the $K_{L}-K_{S}$ mass difference, the $K_{L} \rightarrow \gamma \gamma$ and the Dalitz decays of $K_{L}$ [7].

Confirmation of the BABAR resonance as a four-quark meson will open a new window of hadron physics.

\section{ACKNOWLEDGMENTS}

The author would like to thank Professor T. Onogi for providing information of the BABAR resonance, discussions and encouragements, and Professor T. Kunihiro for discussions and encouragements. He also would like to appreciate Dr. C. Everton for careful reading of the manuscript.
[1] BABAR Collaboration, B. Aubert et al., Phys. Rev. Lett. 90, 242001 (2003).

[2] CLEO Collaboration, D. Besson et al., hep-ex/0305017.

[3] R.L. Jaffe, Phys. Rev. D 15, 267 (1977); 15, 281 (1977).

[4] K. Terasaki and S. Oneda, Phys. Rev. D 38, 132 (1988).

[5] K. Terasaki and S. Oneda, Phys. Rev. D 47, 199 (1993).

[6] K. Terasaki, Phys. Rev. D 59, 114001 (1999).

[7] K. Terasaki, Int. J. Mod. Phys. A 16, 1605 (2001) and references quoted therein.

[8] S. Okubo, Phys. Lett. 5, 165 (1963); G. Zweig, CERN Report No. TH401, 1964; J. Iizuka, K. Okada, and O. Shito, Prog. Theor. Phys. 35, 1061 (1965).

[9] H.J. Lipkin, Phys. Lett. 70B, 113 (1977).

[10] M. Suzuki and S.F. Tuan, Phys. Lett. 133B, 125 (1983).
[11] T. Barnes, F.E. Close, and H.J. Lipkin, hep-ph/0305025.

[12] A.P. Szczepaniak, hep-ph/0305060.

[13] H.-Y. Chen and W.-S. Hou, hep-ph/0305038.

[14] R.N. Cahn and J.D. Jackson, hep-ph/0305012.

[15] M.A. Nowak, M. Rho, and I. Zahed, Phys. Rev. D 48, 4370 (1993); W.A. Bardeen and C.T. Hill, ibid. 49, 409 (1994).

[16] S. Godfrey, hep-ph/0305122.

[17] G. Bali, hep-ph/0305209.

[18] S. Oneda and K. Terasaki, Prog. Theor. Phys. Suppl. 82, 1 (1985).

[19] T. Feldmann, Int. J. Mod. Phys. A 15, 159 (2000).

[20] Particle Data Group, K. Hagiwara et al., Phys. Rev. D 66, 010001 (2002). 